\title{
MIRINGOPLASTIA POR TÉCNICA OVERLAY VERSUS UNDERLAY: VALORACIÓN DEL ÉXITO DE LA CIRUGÍA POR ESTUDIOS MORFOLÓGICOS Y FUNCIONALES EN PACIENTES OPERADOS EN EL HOSPITAL MILITAR DE GUAYAQUIL-ECUADOR 2000-2011
}

\author{
MYRINGOPLASTY BY OVERLAY VERSUS UNDERLAY TECHNIQUE: ASSESSMENT OF THE SUCCESS \\ OF THE SURGERY BY MORPHOLOGICAL AND FUNCTIONAL STUDIES IN PATIENTS OPERATED ON IN \\ THE MILITARY HOSPITAL OF GUAYAQUIL-ECUADOR 2000-2011
}

\author{
TÉCNICA DE MIRINGOPLASTIA POR OVERLAY VERSUS UNDERLAY: AVALIAÇÃO DO SUCESSO DA CIRURGIA \\ POR MEIO DE ESTUDOS MORFOLÓGICOS E FUNCIONAIS EM PACIENTES OPERADOS NO HOSPITAL MILITAR DE \\ GUAYAQUIL-EQUADOR 2000-2011
}

\section{HUMBERTO ESPINOZA ASTUDILLO', ANA PAULA ESPINOZA JOSÉ², CYNTHIA RODRÍGUEZ BOSQUEZ2', MARÍA RAQUEL PÉREZ GONZÁLEZ ${ }^{3}$}

${ }^{1}$ Universidad Católica de Santiago de Guayaquil, Ecuador
${ }^{2}$ Clínica Kennedy, Guayaquil, Ecuador
${ }^{3}$ Coordinación Provincial de prestadores del Guayas, Ecuador

RESUMEN

Introducción: la otitis media crónica es una de las principales causas de perforación timpánica. El tratamiento quirúrgico de primera elección para los casos persistentes de perforación timpánica es la timpanoplastia. Se han desarrollado varias técnicas quirúrgicas, como colocar el injerto por debajo del remanente timpánico (underlay), sobre el mismo (overlay) o una combinación de las dos. Objetivo: determinar cuál es la técnica quirúrgica (Overlay o Underlay) con una mejor tasa de éxito, analizando el cambio morfológico y funcional del posoperatorio. Metodología: estudio de cohorte retrospectivo realizado desde el año 2000 al 2011, en el hospital Militar de Guayaquil. Se incluyeron 72 pacientes de ambos géneros entre 5-60 años. Se excluyó aquellos con hipoacusia congénita, hipoacusia por enfermedades como: diabetes mellitus, hipoacusia neurosensorial y pacientes con cirugías de oído previas. Se obtiene como parámetro de medición audiológica el PTA (promedio de notas puras)y otoscopias posoperatorias para la valoración funcional y morfológica respectivamente. Se analizó la muestra mediante T de student y Kolmogorov para indicar la normalización de los datos con significancia de $p<0.05$ y chi cuadrado para las tablas cruzadas. Resultados: con la técnica Underlay se obtuvo mejor tasa de éxito audiológico con $77.14 \%$, versus Overlay: $18.92 \%, \mathrm{p}<0.0001$. La tasa de reepitelización no varió mucho entre ambas técnicas: Underlay: 88.57\%, Overlay: 86.49\%, $\mathrm{p}=0.79$. El génerono influyó en el éxito terapéutico; Femenino 29, Masculino 20, $(\mathrm{p}=0.3)$. Discusión: se obtuvieron mejores resultados audiológicos con la técnica Underlay sobre la técnica Overlay (GAP $<10 \mathrm{DB}$ ), aunque la reepitelización y el cierre de la perforación sus resultados fueronno significativos. PALABRAS CLAVE: timpanoplastia, perforación de membrana timpánica, otitis media crónica.

ABSTRACT

Introduction: chronic otitis media is one of the principal causes of tympanic perforation. The first-line surgical treatment for persistent cases of eardrum perforation is tympanoplasty. Various surgical techniques have been developed, such as placing the graft below the tympanic remnant (underlay), over it (overlay), or a combination of the two. Objective: determine which is the surgical technique (Overlay or Underlay) with a better success rate, analyzing the morphological and functional change in a postoperative period. Methodology: retrospective cohort study carried out from 2000 to 2011 at the Military Hospital of Guayaquil. Seventy-two patients of both genders between 5-60 years were included. Those with congenital hearing loss, hearing loss due to diseases such as diabetes mellitus, sensorineural hearing loss, and patients with previous ear surgeries were excluded. The audiological measurement parameter is obtained as the PTA (Pure Tone Average) and postoperative otoscopies for the functional and morphological evaluation respectively. The sample was analyzed using T-Student and Kolmogorov to indicate the normalization of the data with the significance of $\mathrm{p}<0.05$ and chi-square for the crossed tables. Results: with the Underlay technique, a better audiological success rate was obtained with $77.14 \%$, versus 0 verlay: $18.92 \%, p<0.0001$. The re-epithelialization rate did not vary much between the two techniques: Underlay: $88.57 \%$, Overlay: $86.49 \%, p=0.79$. Gender did not influence therapeutic success; Female 29, Male 20, $(p=0.3)$. Discussion: better audiological results were obtained with the Underlay technique over the Overlay technique $(\mathrm{PTA}<10 \mathrm{DB})$, although the re-epithelialization and the closure of the perforation were not significant. KEYWORDS: tympanoplasty, tympanic membrane perforation, chronic otitis media.

Introdução: a otite média crônicaé uma das principais causas de perfuração timpânica. Otratamento cirúrgico de primeira linha para casos persistentes de perfuração do tímpano éa timpanoplastia. Várias técnicas cirúrgicas foram desenvolvidas, como a colocação do enxerto abaixo do remanescente timpânico (underlay), sobre ele (overlay) ou uma combinação dos dois. Objetivo: determinar qual é a técnica cirứrgica (Overlay ou Underlay) com melhor índice de sucesso, analisando a alteração morfológica e funcional pós-operatória. Metodologia: estudo de coorte retrospectivo realizado de 2000 a 2011, no hospital Militar de Guayaquil. Setenta e dois pacientes de ambos os sexos entre 5-60 anos foram incluídos. Foram excluídos aqueles com perda auditiva congênita, perda auditiva decorrente de doenças como: diabetes mellitus, hipoacusia neurossensorial e pacientes com cirurgias otológicas anteriores. O parâmetro de medida audiológica é obtido como PTA (média dos escores puros)e otoscopias pós-operatórias para avaliação funcional e morfológica respectivamente. A amostra foi analisada pelo Tde Student e Kolmogorov para indicar a normalização dos dados com significância de $p<0,05$ e qui quadrado para as tabelas cruzadas. Resultados: com a técnica Underlay, obteve-se melhor taxa de sucesso audiológico com 77,14\%, versus Overlay: $18,92 \%, \mathrm{p}<0,0001$. A taxa de reepitelização não variou muito entre as duas técnicas: Underlay: 88,57\%, Overlay: 86,49\%, $p=$ 0,79. 0 gênero não influenciou o sucesso terapêutico; Feminino 29, Masculino 20, ( $p=0,3)$. Discussão: melhores resultados audiológicos foram obtidos com a técnica Underlay sobre a técnica Overlay (GAP<10 DB), embora a reepitelização e o fechamento da perfuração não tenham sido significativos. PALAVRAS-CHAVE: timpanoplastia, perfuracao membrana timpánica, otite média crónica. 


\section{INTRODUCCIÓN}

La otitis media crónica (OMC) es una de las principales causas de hipoacusia conductiva en la población pediátrica y adulta especialmente en países en vía de desarrollo como Ecuador. 1,3,5

Según La Organización Mundial de la Salud, la OMC afecta a $0.5-43 \%$ de la población mundial; actualmente 20 millones de personas padecen de esta patología de los cuales el $60 \%$ podría ser reversible". ${ }^{2}$ Se estima que Sudamérica es la cuarta región con mayor prevalencia de OMC. ${ }^{3}$

Las infecciones recurrentes, el trauma auditivo y la iatrogenia médica son una de las principales causas de perforación timpánica siendo las infecciones las de mayor prevalencia. ${ }^{4,9,17} \mathrm{La}$ OMC no tratada puede producir en algunos casos serias complicaciones, como son abscesos cerebrales, meningitis, ${ }^{29}$ mastoiditis, hipoacusia crónica irreversible y en la población pediátrica, alteración en el desarrollo cognitivo e incluso la muerte. $3,5,7,23,24,30$

El tratamiento definitivo para la perforación timpánica (luego de haber tratado la infección en caso de que exista) es la cirugía reconstructiva y cierre de la misma, llamada timpanoplastia, ${ }^{5}$ cuyo objetivo principal es erradicar la enfermedad del oído medio, restablecer la integridad de la membrana timpánica y por ende la recuperación audiológica. ${ }^{6,7,9,13}$

Estudios previos han demostrado que la tasa de éxito de la misma es del 93\%-95\%, ${ }^{2}$ pero que ésta varía según el tipo de injerto utilizado, el tiempo transcurrido desde cuando se presenta hasta la intervención y la técnica quirúrgica empleada. ${ }^{8,9}$

Se han desarrollado un sin número de técnicas quirúrgicas, agrupadas en dos: Overlay (colocación del injerto lateral al annulus timpánico) y underlay (colocación medial al annulus timpánico) o una combinación de ellas. ${ }^{10-13}$ Sin embargo, diferentes estudios reportan tasas de éxitos, reepitelización, ganancia y complicaciones diferentes para cada una de las técnicas. ${ }^{4,6,9,11,14}$

El objetivo principal de este estudio es determinar cuál es la mejor técnica quirúrgica de timpanoplastia, sea esta Overlay o Underlay, en la que se obtiene una mayor tasa de éxito, analizando el cambio morfológico y funcional que presentan los pacientes en el posoperatorio. (Figura 1, 2 y 3).

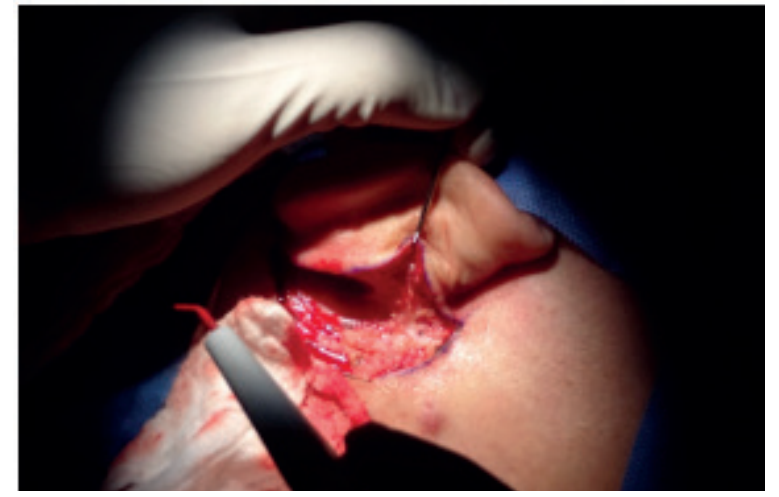

Figura 1. Incisión retroauricular, con obtención de fascia de músculo temporal como material de injerto.

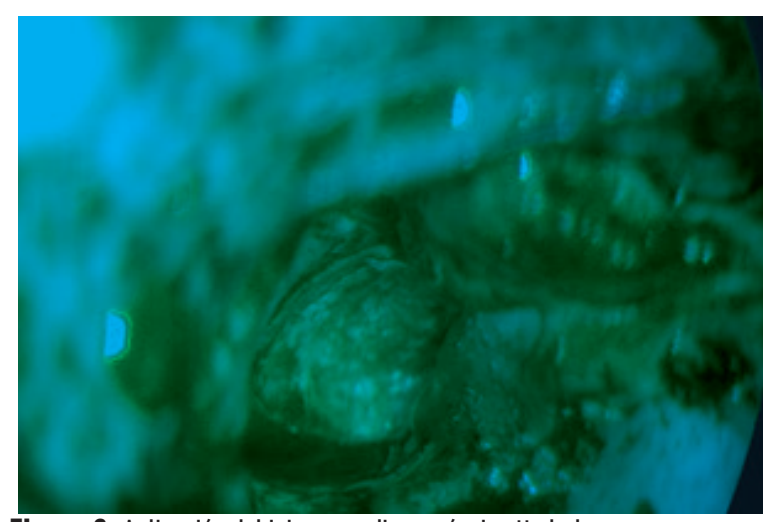

Figura 2. Aplicación del injerto mediante técnica Underlay.

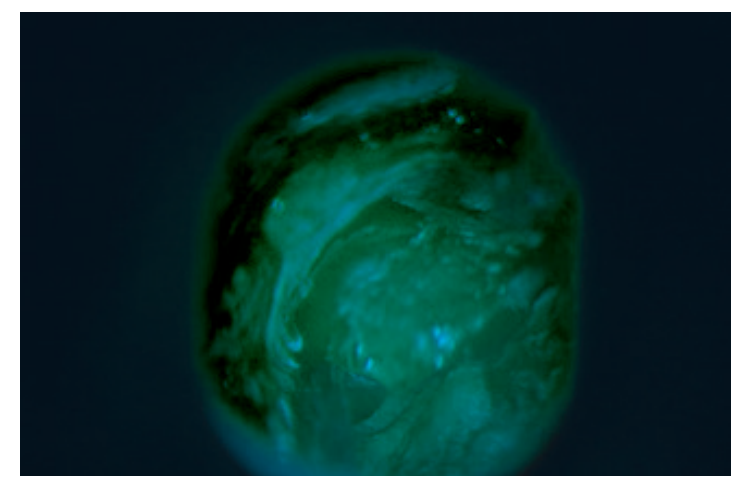

Figura 3. Aplicación del injerto mediante técnica Overlay.

\section{METODOLOGÍA}

Se realizó un estudio de cohorte retrospectivo comprendido entre los años 2000-2011. Para la recolección de datos se realizóla revisión autorizada decada historia clínica emitida por el departamento de estadística del hospital Militar. Se tabularon los datos de los pacientes en hojas de cálculos de EXCEL versión 19.0.

Se incluyeron 72 pacientes de ambos géneros cuyas edades oscilaban entre 5-60 años de edad intervenidos quirúrgicamente de timpanoplastia por OMC simple, que solo hayan sido sometidos a dicha intervención quirúrgica en una ocasión 
durante la evolución de su enfermedad y aquellos en cuya historia clínica debían tener registrado una audiometría tonal previa y posterior a la intervención durante los años 2000-2011.Se excluyó a pacientes con déficit audiológico congénito, con enfermedades generales causantes de hipoacusia como diabetes mellitus y otras afecciones, con sordera neurosensorial y en quienes además de la timpanoplastia se hayan realizado otro tipo de cirugía de oído como por ejemplo mastoidectomía.

Se analizaron las siguientes variables: 1) edad: expresada en años y dividida en 3 rangos (0-20; 21-40, 41-60); 2) Género: femenino o masculino; 3) ACPTA (ponerla en intro) (Air Conduction Pure Tone Average) prequirúrgico: definido como el promedio de decibeles en las frecuencias 500, $1000,2000 \mathrm{~Hz}$ de la vía aérea de las audiometrías prequirúrgicas; 4) BCPTA (Bone Conduction Pure Tone Average) prequirúrgico definido como el promedio de decibeles en las frecuencias 500, 1000, $2000 \mathrm{~Hz}$ de la vía ósea de las audiometrías prequirúrgicas; 5) ABGPTA (Air Bone Gap Pure Tone Average) prequirúrgico: expresada en decibeles, definido como la diferencia entre la vía aérea y ósea en las frecuencias 500,1000, $2000 \mathrm{~Hz}$ de las audiometrías prequirúrgicas; 6) ACPTA (Air Conduction Pure Tone Average) posquirúrgico: definido como el promedio de decibeles en las frecuencias 500, 1000, $2000 \mathrm{~Hz}$ de la vía aérea de las audiometrías posquirúrgicas; 7) BCPTA (Bone Conduction Pure Tone Average) posquirúrgico definido como el promedio de decibeles en las frecuencias 500, 1000, $2000 \mathrm{~Hz}$ de la vía ósea de las audiometrías postquirúrgicas; 8) ABGPTA (Air Bone Gap Pure Tone Average) posquirúrgico: expresada en decibeles, definido como la diferencia entre la vía aérea y ósea en las frecuencias 500, 1000, $2000 \mathrm{~Hz}$ de las audiometrías posquirúrgicas;, 9) Reepitelización: definido como la ausencia de perforación timpánica determinado por otoscopia endoscópica o por microscopía; 10) Tipo de técnica quirúrgica: Underlay o Overlay; 11) Timpanoplastia exitosa (variable dicotómica): se definió como a aquellas cuyo $\mathrm{ABGPTA}$ posquirúrgico fue menos o igual a $10 \mathrm{~dB}$ (audición normal: $\leq 10 \mathrm{~dB}$, hipoacusia leve: entre 11-20 dB, hipoacusia moderada: 21-30 dB, hipoacusia severa:> $30 \mathrm{~dB})^{4,9}, 12$ ) Complicaciones (variable dicotómica): definida como la presencia o no de complicaciones tales como: rechazo del injerto, estenosis del conducto auditivo externo, quiste epitelial, granuloma, reperforación de la membrana timpánica, retracción timpánica, colesteatoma, infección y blunting. 13) Ganancia: expresada en decibeles, definido como la diferencia entre el ACPTA prequirúrgico del ACPTA posquirúrgico, entre el $\mathrm{BCPTA}$ prequirúrgico del $\mathrm{BCPTA}$ posquirúrgico, y entre el $\mathrm{ABCPTA}$ prequirúrgico del ABGPTA posquirúrgico.

Elanálisis estadístico se realizó con los programas MINI TAB y MedCALC. Para determinar la distribución de las edades y de ACPTA, BCPTA, AGPTA se utilizó el test de Kolmogorov-Smirnov, quecomprobóla distribución normal delas mismas lo que permitió el análisis con test de student a dos colas. Las variables categóricas fueron analizadas con prueba chi cuadrado. Se consideró $\mathrm{p}<0.05$ como estadísticamente significativo.

RESULTADOS

Serealizóelestudiocon una muestra de 72 pacientes, de los cuales 38 ( $52.78 \%$ ) eran hombres y mujeres 34 (47.22\%). La edad media fue 26.04 años. La tasa de éxito audiológico de las cirugías fue de $80.5 \%$ $(\mathrm{N}=58)$. La tasa de reepitelización exitosa de las cirugías fue $87.5 \%(\mathrm{~N}=63)$. La complicación más común fue la reperforación timpánica 9 (12.5\%) seguido de la estenosis del conducto auditivo externo 4 (5.56\%) y las infecciones 4 (5.56\%.).

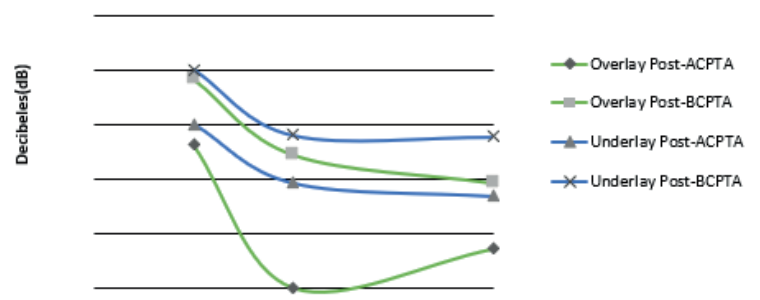

UNDERLAY+

Esta técnica resultó exitosa en 77.14\% (27) de los casos (éxito = ABGPTA postquirúrgico $\leq 10 \mathrm{~dB}$ ); 5 (14.29\%) sí presentaron mejoría auditiva pero no en rangos de éxito y $3(8.57 \%$ ) pacientes presentaron deterioro auditivo posquirúrgico (Tabla 1).

TABLA 1. PROMEDIO DE NOTAS PURAS (PTA) PRE-POSQUIRÚRGICO EN UNDERLAY Y OVERLAY

\begin{tabular}{rlrrr} 
& PTA (dB) & Underlay & Overlay & Valor $\mathbf{p}$ \\
\hline \multirow{2}{*}{ ACPTA } & Prequirúrgico & $42.18 \pm 16.04$ & $41.99 \pm 14.40$ & 0.96 \\
\cline { 2 - 5 } & Posquirúrgico & $27.92 \pm 9.53$ & $38.96 \pm 14.30$ & 0.0003 \\
\hline \multirow{2}{*}{ BCPTA } & Prequirúrgico & $22.66 \pm 11.69$ & $22.44 \pm 10.77$ & 0.93 \\
\cline { 2 - 5 } & Posquirúrgico & $18.00 \pm 7.98$ & $22.59 \pm 9.76$ & 0.033 \\
\hline \multirow{2}{*}{ ABGPTA } & Prequirúrgico & $16.99 \pm 10.84$ & $19.72 \pm 10.06$ & 0.27 \\
\cline { 2 - 5 } & Posquirúrgico & $9.35 \pm 6.13$ & $17.28 \pm 8.42$ & $<0.0001$
\end{tabular}

Es destacable la evolución del ABGPT. El ABGPTAprequirúrgico (media) fue $16.99 \mathrm{~dB}: \mathrm{ABCPTA} \leq 10$ 
dB: 11 (31.43\%); ABGPTA entre 11 y $20 \mathrm{~dB}: 14$ (40\%); ABGPTA entre 21 y $30 \mathrm{~dB}: 4$ (11.43\%); ABGPTA mayor a $30 \mathrm{~dB}: 6(17.14 \%)$. El ABGPTA posquirúrgico (media) fue $9.35 \mathrm{~dB}$ : ABGPTA $\leq 10 \mathrm{~dB}: 27$ (77.14\%); ABGPTA entre 11 y $20 \mathrm{~dB}: 6(17.14 \%)$; ABGPTA entre 21 y 30 dB: 1 (2.86\%); ABGPTA > 30 dB: 1(2.86\%). Se demostró una reducción significativa del gap entre la vía aérea y la ósea (ABGPTA prequirúrgico:

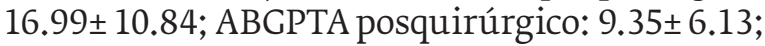
$\mathrm{p}=0.0005)$; además de una reducción significativa de la vía aérea

(ACPTA prequirúrgico: 42.18 \pm 16.04 ; ACPTA posquirúrgico: 27.92ะ 9.39; $\mathrm{p}<0.00001$ ); sin embargo, la reducción en la vía ósea no fue significativa (BCPTA prequirúrgico: $22.66 \pm 11.19$, BCPTA posquirúrgico: $18 \pm 7.98 ; \mathrm{p}=0.06)$. La tasa de reepitelización exitosa fue de $88.57 \%$ (30), La tasa de complicación fue 10 (28.57\%), siendo la más común la reperforación timpánica seguida de colesteatoma (Tabla 4).

TABLA 4. ÉXITO MORFOLÓGICO Y FUNCIONAL DE TÉCNICA UNDERLAY Y OVERLAY

\begin{tabular}{llll}
\multicolumn{4}{c}{ No. casos (\%) } \\
& Underlay & Overlay & Valor p \\
\hline Éxito audiológico & $27(77.14)$ & $7(12.92)$ & 0.0001 \\
\hline Reepitelización exitosa & $31(88.57)$ & $32(86.49)$ & 0.79
\end{tabular}

TABLA 3. COMPLICACIONES POSTERIORES A TIMPANOPLASTIA OVERLAY Y UNDERLAY

\begin{tabular}{|c|c|c|c|}
\hline \multicolumn{4}{|c|}{ NO. DE CASOS (\%) } \\
\hline & Complicaciones & Underlay & Overlay \\
\hline & Valor $\mathrm{P}$ & & \\
\hline Infecciones & $1(2.86)$ & $3(8.11)$ & - \\
\hline Rechazo injerto & $0(0.00)$ & $1(2.70)$ & - \\
\hline Estenosis CAE & $1(2.86)$ & $3(8.11)$ & - \\
\hline Quiste epitelial & $0(0.00)$ & $2(5.41)$ & - \\
\hline Granuloma & $1(2.86)$ & $0(0.00)$ & \\
\hline Reperforación & $4(11.43)$ & $5(13.51)$ & \\
\hline Retracción & $1(2.86)$ & $0(0.00)$ & \\
\hline Colesteatoma & $2(5.71)$ & $0(0.00)$ & \\
\hline TOTAL & $10(28.57)$ & $14(37.84)$ & \\
\hline
\end{tabular}

*CAE: conducto auditivo externo.

OVERLAY

La técnica Overlay resultó exitosa en $18.92 \%$ (7) de los casos (éxito = ABGPTA posquirúrgico $\leq 10$ dB); 25 (67.57 \%) sí presentaron mejoría auditiva pero no en los rangos predeterminados como exitoso y $5(13.51 \%)$ pacientes presentaron deterioro auditivo posquirúrgico. Es destacable la evolución del ABGPT. El ABCPTA prequirúrgico (media): 19.72
$\mathrm{dB}: \mathrm{ABGPTA} \leq 10 \mathrm{~dB}: 7$ (18.92\%); ABGPTA entre 11 y $20 \mathrm{~dB}$ : 14 (37.84\%); ABGPTA entre 21 y $30 \mathrm{~dB}: 9$ (24.32\%); ABGPTA > $30 \mathrm{~dB}: 7(18.92 \%)$. El ABGPTA posquirúrgico (media) fue 17.28: ABGPTA $\leq 10 \mathrm{~dB}$ : 7 (18.92\%); ABGPTA entre 11 y $20 \mathrm{~dB}: 19$ (51.35\%); ABCPTA entre 21 y $30 \mathrm{~dB}: 8$ (21.62\%); ABGPTA >30dB: $3(8.11 \%)$

No se demostró una reducción significativa en ningún PTA. ABGPTA prequirúrgico: 19.72 \pm 10.06 , ABGPTA posquirúrgico: $17.28 \pm 8.42$, $\mathrm{p}=0.26$; $\mathrm{ACPTA}$ prequirúrgico: $41.99 \pm 14.40$, АCРTA posquirúrgico: $38.96 \pm 14.30, \mathrm{p}=0.37$; $\mathrm{BCPTA}$ prequirúrgico: $22.44 \pm 10.77$, BCPTA posquirúrgico: $22.59 \pm 9.76$, $\mathrm{p}=0.95$. La tasa de reepitelización exitosa fue 86.40\%(32). La tasa de complicación fue 14 (37.84\%), siendo la más común la reperforación timpánica seguida de estenosis del conducto auditivo externo e infecciones (Tabla 3).

\section{AMBAS}

Los PTA prequirúrgicos entre ambas técnicas no variaron significativamente(Tabla 1). Sin embargo, los PTA posquirúrgicos entre ambas técnicas sí variaron significativamente, especialmente el ABGPTA (ABGPTA posquirúrgico Underlay: 9.35 \pm 6.13 , Overlay: $17.28 \pm 8.42$, $\mathrm{p}<0.0001$ ) (Figura 4).

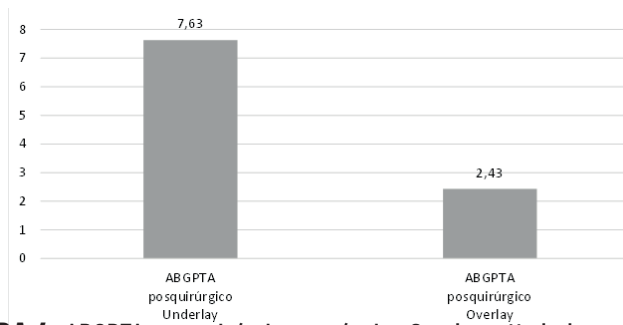

FIGURA 4. ABGPTA postquirúrgico en técnica Overlay y Underlay.

En cuanto a la ganancia, todos los PTA resultaron estadísticamente significativos (Tabla 2). El género no influyó en el éxito terapéutico; F: 29, M: 20, $(\mathrm{p}=0.3)$. La edad no influyó en el éxito terapéutico: 0-20 años: 22 (38.56\%), 21-40: 28 (38.89\%), 41-60:8 $(11.11 \%) \mathrm{p}=0.39$. No hubo diferencia significativa de las complicaciones entre ambas técnicas quirúrgicas: Overlay 14 (37.84\%), Underlay 10 (28.57), $\mathrm{p}=0.4$.

TABLA 2. GANANCIA DE PROMEDIO DE NOTAS PURAS CON TÉCNICA UNDERLAY Y OVERLAY

\begin{tabular}{lrcr}
\multicolumn{4}{c}{ Técnica quirúrgica Media (dB) +- Desviación Standard } \\
\hline Ganancia & Underlay & Overlay & Valor P \\
\hline ACPTA & $14.26 \pm 14.77$ & $3.03 \pm 8.84$ & 0.0002 \\
\hline BCPTA & $4.67 \pm 11.3$ & $0.15 \pm 8.74$ & 0.04 \\
\hline ABGPTA & $7.63 \pm 9.98$ & $2.43 \pm 9.88$ & 0.02 \\
\hline
\end{tabular}

ACPTA: air conduction pure tone average; BCPTA: bone conduction pure tone average; ABGPTA: air bone gap pure tone average. 
DISCUSIÓN

Ambas técnicas quirúrgicas de timpanoplastia producen una mejoría en la audición. La técnica Underlay mostró ser superior que la Overlay para reducir el gap entre la vía aérea y la ósea $(\mathrm{p}=<0.0001)$, y además mostró ser superior que la Overlay para producir éxito terapéutico $(\mathrm{p}=0.0001)$. Con ambas técnicas quirúrgicas se produce reepitelización exitosa sin una diferencia estadísticamente significativa $(p=0.79)$. La tasa de complicaciones fue muy parecida entre ambas técnicas y se presentó en muy pocos casos. Factores como el género y edad no influyeron significativamente en los resultados. ${ }^{19}$

Aligualquelodemuestra la bibliografía consultada, ambas técnicas quirúrgicas lograron una ganancia auditiva, es decir reducir el GAP pre-operatorio.,18,27 Esto concuerda con los resultados de Rizer et al ${ }^{18}$ 1997 que reportó ganancia auditiva en el 92.8\% de los pacientes intervenidos con underlay vs $57.1 \%$ Overlay. Sin embargo, en el presenteestudio a pesar de que ambas técnicas contribuyeron a la ganancia auditiva de los pacientes, es necesario destacar que previo a la cirugía la gran mayoría de los pacientes intervenidos con Underlay presentaban hipoacusia leve y luego de esta la gran mayoría tuvo audición normal, no siendo asícon la técnica Overlay ya que la gran mayoría presentaban hipoacusia leve y se mantuvieron así posterior a la cirugía.

La tasa de éxito (ABGPTA $\leq$ a $10 \mathrm{~dB}$ ) conseguida con la técnica Underlay fue de $77.14 \%$ mientras que con la técnica Overlay fue de $18,92 \%$ ( $\mathrm{p}=0.0001$ ). En un estudio similar de 115 casos, B. Sergi et al mostraron $63,2 \%$ (31) casos exitosos con técnica Underlay mientras que 29,6\% (16) casos exitosos con técnica Overlay.4 Glasscock reportó tasas de éxito de $96 \%$ usando la Técnica Underlay y $91 \%$ con Overlay y, en un total de 273 participantes". ${ }^{14,16}$ Rizer reportó en su estudio tasas de éxito de 95.6\% en 554 injertos con técnica Overlay, mientras que solo $88.8 \%$ en 158 injertos con técnica Underlay. ${ }^{14}$ Nuestro estudio concuerda con los resultados descritos por Doyle ${ }^{18}$ y por Glasscock, ${ }^{18}$ pero no así con los de Rizer. ${ }^{18}$ Concordamos con la conclusión de Segi et al 2011:4 "El alto éxito alcanzado con la técnica underlay es probablemente a la reducida manipulación quirúrgica de estructuras y a una curación más rápida que minimiza el riesgo de fallas y por ello menos pérdida del injerto”.

Ambas técnicas quirúrgicas mostraron producir reepitelización de la membrana timpánica sin una diferencia estadísticamente significante $(\mathrm{p}=$
0.79). Mangal et al 2003, ${ }^{17}$ encontró una tasa de reepitelización exactamente iguales (93.3\%) para ambas técnicas en un estudiode60 pacientes. Doyle et al. $1972^{16}$ mostraron una tasa de reepitelización de $88 \%$ para Underlay, $57 \%$ Overlay en un estudio de 131 pacientes. Los resultados de este trabajo coinciden con los de Mangal et al. ${ }^{17}$ Por lo tanto, es alentador no usar la tasa de reepitelización como criterio de preferencia de una técnica sobre la otra. Los resultados obtenidos demostraron que la complicación más frecuente fue la reperforación timpánica tanto para underlay como para overlay. En la bibliografía mencionan que las infecciones y la reperforación como complicaciones frecuentes para underlay, $, 4,6,18,20$ lo cual concuerda con el presente estudio.

Sin embargo, en otras publicaciones se destaca como complicaciones constantes de Overlay: lateralización del injerto, colesteatoma iatrogénico yblunting, $, 4,18,20$ las mismas que no se presentaron en este estudio. La tasa de complicaciones fue similar entre ambas técnicas, no significativo $(\mathrm{p}=0.404)$, por lo tanto, el apoyo a que la tasa de complicaciones no sea utilizada como criterio para determinar cuál técnica quirúrgica es superior. Una de las principales fortalezas de este estudio es que involucró pacientes intervenidos quirúrgicamente en un periodo de once años, tiempo ideal para analizar el desarrollo de complicaciones postoperatorias. Evidentemente es una ventaja el acceso a las historias clínicas y contar con datos precisos de cada paciente.

Sin embargo, sí existen ciertos factores que pudieron influenciar negativamente en el estudio, como por ejemplo el tiempo que transcurre desde que el paciente tiene la lesión hasta que finalmente es intervenido quirúrgicamente. Aun cuando la cadena oscicular parezca intacta, es probable que haya fibrosis y de hecho timpanoesclerosis lo cual interferiría en los resultados posoperatorios. A medida que pase más tiempo, es razonable pensar que haya mayor probabilidad de tener más fibrosis, por la inflamación subsecuente, debidos a períodos de reagudización de su otorrea por infección. ${ }^{18}$

La causa de la perforación timpánica también podría interferir en los resultados ya que si la perforación fue producida por infecciones recurrentes debido a otitis media crónica habrá mayor riesgo de cambios inflamatorios mientras que la producida por trauma acústico habrá menor riesgo, y por lo tanto la tasa de reepitelización podría estar afectada. ${ }^{18}$ Entre las restricciones 
constan que, debido a la naturaleza retrospectiva del estudio, nos basamos en datos previamente recolectados siendo esta una posibilidad de sesgo de información.

Además, la ausencia deestudios clínicos quevaloren la incidencia y prevalencia de la OMC y perforación timpánica enEcuador específicamente, dificultenel desarrollo de muchos estudios adicionales y debido a ello es que se propone en nuestro país se realicen estudios de investigación sobre estos temas que tendrían una gran importancia epidemiológica. Otra segunda sugerencia es que se realicen estudios en nuestro país en donde se analicen la técnica quirúrgica más conveniente según el tipo de lesión que tenga cada paciente como una perforación timpánica anterior o posterior, grande o pequeña para así determinar si realmente existe una diferencia en otros aspectos sobre ambas técnicas. ${ }^{26,28}$

CONCLUSIÓN

Se concluye que el uso de la técnica Underlay para la timpanoplastia simple sobre la Overlay por la mayor tasa de éxitos de cierre de perforación timpánica, así como disminuir el GAP y llevar la audición a valores normales (GAP <10 DB).

\section{REFERENCIAS BIBLIOGRÁFICAS}

1. Castaño R. Otitis media crónica como problema de salud publico en países en vía de desarrollo. Acta Otorrinolaringológica de Colombia. 2001; 29(3):1,3.

2. Pérez M., Torres J. Eficacia terapéutica de la timpanoplastia transcanal con injerto del cartílago y pericondrio. Rev.Fac. Med., Bogotá. 2007Jul 19, 55(3):146-151.

3. World Health Organization. Child and adolescent health development. Prevention of blindness and deafness. Chronic suppurative otitis media. Burgen of illness and Management options. Geneva. Switzerland; 2004.

4. Sergi B. GalliJ, De corso E, Parrilla C, Paludetti G. Overlay versus underlay myringoplasty: report of outcomes considering closure of perforation and hearing function. Acta otorhinolaryngologica Italica. 2011; 31:366-371.

5. Mora E. Manual CTO de medicina y cirugía. Otorrinolaringologia.8vaed. Madrid: CTOeditorial; 2011.

6. Shaikh A., et al. Outcome of Tympanoplasty type. 1 by Underlay technique. JLUMHS. 2009; 8(1):80-84.
7. Boerninghaus $\mathrm{H}$. Otorrinolaringología para estudiantes de medicina. 9nd ed. Barcelona: Spinger-Velorg Iberica; 1995.

8. Kageyama A. Factores de riesgo en el fracaso dela miringoplastia. GacMedMex. 2001; 137(3):209-220.

9. Maroto D.et al. Resultado funcional de las miringoplastias. Acta otorrinolaringol Esp.2010; 61(2):94-99.

10. Kartush J. etal. Over-Under Tympanoplasty. The laryngoscope.2002; 112:802-807.

11. Yigit O. etal. Short-term evaluation of over-under myringoplasty technique. Eur Archotorrinolaryngol. 2005; 262:400-403.

12. Lee HY.et al. Loop overlay Tympanoplasty for anterior or subtotal perforations. Auris Nasus Larynx. 2010; 37:162, -166.

13. Ryan J, Briggs R. Outcomes of the overlay graft technique in Tympanoplasty. ANZ J Surg. 2010; 80:624-629.

14. Haberman R. Middle ear and mastoid surgery. 1ra Ed. New York: Thieme editorial; 2004.

15. British society of audiology. Pure tone air and bone conduction threshold audiometry with and without masking. 2011.

16. Rizer F. Overlay versus underlay Tympanoplasty. Part I: Historical review of the literature. Laryngoscope. 1997;107(12 pt 2):1-25

17. Mangal S. Comparative study of the underlay and overlay techniques of myringoplasty in large and subtotal perforations of the tympanic membrane. The journal of laryngology and Otology. 2003; 117:444-448

18. RizerF. Overlay versus underlay Tympanoplasty: Part II the study. Laryngoscope. 1997; 107:26-36.

19. Prakash M. Prospective study of 100 cases of underlay Tympanoplasty with superiorly based circumferential flap for subtotal perforations. Indian journal of otolaryngology and Head and Neck Surgey. 2007; 59(3):225.228.

20. Adkins W. Tympanoplasty; influencing factors. The Laryngoscope.2009; 94 (7):916-918.

21. Sengupta A. A study outcome of Underlay Overlay and combined techniques of Myringoplasty. Indian J Otolaryngology Head and a Neck Surg. 2012 Marzo; 64(1): 63-66

22. Glasscock-Shambough. Surgery of the ear. 6ta edition. People Medical Publishing House USA Editorial; 2003: pp 401-403.

23. Kangsanarak J. Intracranial complications of suppurative otitis media: 13 years experience. 1995. January; 16(1):104-109.

24. Lin Y.et al. The prevalence of chronic otitis media and its complication rates in teenagers and adult 
patients. Otolaryngol Head and Neck Surg. 2009 Feb; 140(2):165-170.

25. Shu M. "Epithelial pearl” after Tympanoplasty with the underlay technique. Ear nose Throat J. 2011 May: 90(5):206.

26. Lee P, Kelly G, Mills RP. Myringoplasty: does the size of the perforation matter? . Clin Otolaryngol. Allied Sci. 2002 Oct; 27(5):331-4.

27. Pignataro L, Grillo Della Berta L, Capaccio P, Zaghis A. Myringoplasty in children: anatomical and functional results. J Laryngol Otol. 2001 May; 115(5):369-73.
28. Faramarzi A, Hashemi SB, Rajaee A. "Mucosal pocket" myringoplasty: a modification of underlay technique for anterior or subtotal perforations. Am J Otolaryngol. 2012 Nov-Dec; 33(6):708-13.

29. Ibrahim SI, Cheang PP, Nunez DA. Incidence of meningitis secondary to suppurative otitis media in adults. J Laryngol Otol. 2010 Nov; 124(11):1158-61.

30. Dubey SP, Larawin V. Complications of chronic suppurative otitis media and their management. Laryngoscope. 2007 Feb;117(2):264-7. 